\title{
Prevalence of Mental Health Problems in Children and Its Associated Socio-Familial Factors in Urban Population of Semnan, Iran (2012)
}

\author{
Maryam Seyf Hashemi ${ }^{1}$; Elham Yarian ${ }^{1}$; Parviz Bahadoran ${ }^{2}$; Jafar Jandaghi ${ }^{1}$; Majid \\ Mirmohammad Khani ${ }^{3,4, *}$ \\ ${ }^{1}$ Department of Pediatrics, Semnan University of Medical Sciences, Semnan, IR Iran \\ 2 Oxleas NHS Foundation Trust, London, UK \\ ${ }^{3}$ Department of Epidemiology, Semnan University of Medical Sciences, Semnan, IR Iran \\ ${ }^{4}$ Department of Community Medicine, Research Centre for Social Determinants of Health, School of Medicine, Semnan University of Medical Sciences, Semnan, IR Iran \\ *Corresponding author: Majid Mirmohammad Khani, Department of Community Medicine, Research Centre for Social Determinants of Health, School of Medicine, Semnan Univer- \\ sity of Medical Sciences, Semnan, IR Iran. E-mail: mirmohammadkhani@razi.tums.ac.ir
}

Received: December 29, 2013; Revised: October 8, 2014; Accepted: November 11, 2014

Background:The World Health Organization (WHO)defines mental health as "a state of well-being in which every individual realizes own potential, can cope with the normal pressures of life, is able to work effectively, and can make a contribution to community".

Objectives: Mental Health Problems (MHP) is a great concern for all societies in terms of its burden and impact. This survey screened MHP and its impact in an Iranian urban population aged 6 -12 years old, and explored its associated socio-familial factors.

Patients and Methods: The survey was conducted in the elementary schools of Semnan, using random cluster sampling. Collection and analysis of data was performed using the parent version of the "Strengths and Difficulties Questionnaire(SDQ)" and survey commands of Stata-nine, taking into account cluster effect and population weights. Associations were assessed by fitting simple and multiple logistic regression models. $\mathrm{P}<0.05$ was considered significant.

Results: With regard to the SDQ total score, $19.3 \%$ (95\% CI: 8.6, 30.1) scored above the normal threshold (9.6\% abnormal, 9.7\% borderline). The frequency of problems ranged between 16.1\% (peer problems) and 8.4\% (emotional symptoms), and in all subscales boys were affected more than girls. The impact score was abnormal in $68.4 \%$ of all children, and was greater in girls than in boys. "A previously diagnosed mental health disorder" (OR=11.11, 95\% CI:5.55, 25.00), "male gender" $(\mathrm{OR}=1.43,95 \% \mathrm{CI}: 1.10,1.87$ and "less time spent with the child by father" ( $\mathrm{OR}=1.61,95 \% \mathrm{CI}: 1.20,2.17)$ were significantly associated with an abnormal SDQ.

Conclusions: The high rate of MHP in 6 - 12 year-old children and the lack of any significant correlation with their age, underpins the importance of early screening for MHP in schools, with particular focus on high risk groups.

Keywords: Mental Health; Survey; Child; Population Surveillance; Iran

\section{Background}

The World Health Organization (WHO) defines mental health as "a state of well-being in which every individual realizes own potential, can cope with the normal pressures of life, is able to work effectively, and can make a contribution to community" (1). In today's world maintaining and promoting the mental health of people is a great concern for all societies and imposes a huge financial burden on governments. According to WHO estimates in 2011, approximately $20 \%$ of children and adolescents throughout the world are likely to have some kind of Mental Health Problems (MHP), half of all begin by the age of 14 (1). Failure to identify and treat these disorders will have a substantial adverse impact on children and their families' lives. Mental health conditions experienced by children can lead to higher drop-out rates. Social impairment, along with high rates of juvenile delinquency, substance misuse and suicide are some other notable examples of this impact. Children with sub-clinical mental health conditions (mental health problems not meeting criteria for psychiatric diagnoses) also have poorer educational outcomes (2). In spite of the high rate of MHP among children and adolescents in developing countries, a large number do not receive any intervention for their problems. According to World Mental Health Survey Consortium, 35.5\% to 50.3\% of serious cases in developed countries and $76.3 \%$ to $85.4 \%$ in less-developed countries received no treatment (3). The first step towards any intervention for MHP is finding the extent and frequency of these disorders; and hence in developing countries, including Iran, population-based studies for determining the prevalence of behavioral and emotional problems and their correlates is essential. Considering the high cost of assessing all children for MHP, the use of a valid, simple, quick, and reliable screening tool can greatly expedite the detection of MHP in children and adolescents. This survey was conducted to screen MHP and their impact on children aged 6 - 12 years old (elementary school students) using

Copyright (C) 2015, Growth \& Development Research Center. This is an open-access article distributed under the terms of the Creative Commons Attribution-NonCommercial 4.0 International License (http://creativecommons.org/licenses/by-nc/4.0/) which permits copy and redistribute the material just in noncommercial usages, provided the original work is properly cited. 
the parent version of the "Strengths and Difficulties Questionnaire" (SDQ) in the urban population of Semnan, Iran. Semnan is the provincial capital of the province of Semnan. It is a medium-sized industrial city located in central Iran; approximately 220 kilometers from Tehran with approximately two hundred thousand population Due to the significant socioeconomic and cultural similarities with many other urban areas-especially central and non-border provinces-the city is a good example of an Iranian urban community; and has been selected as a pilot area for several national health projects linked with the social determinants of health by the Ministry of Health.

\section{Objectives}

The study also looked for any family or socioeconomic factors associated with MHP in these children, and finally it studied the impact of these problems on children and their families in Semnan as a representative urban population of the country.

\section{Patients and Methods}

The study was a population based survey conducted in 2012. The target population was children aged 6 - 12 years old, and the study population comprised all children studying in Semnan elementary schools (grades 1 to 6) during the survey. Data were collected using a questionnaire filled out by parents. The study was approved by the Ethics Committee of Semnan University of Medical Sciences. Sample size was set at 1150 after taking into account a conservative 0.5 for the proportion of MHP, allowing the maximum type I error of 5\%, and the design effect of 1.5. A cluster random sampling was used to select subjects. We essentially divided the elementary schools into major groups considering their types (boys' vs. girls' and public vs. private schools). We randomly selected two boys' and two girls' schools from public schools, and one school from each group of private schools ( 6 schools in total). As the average number of students in each classroom was estimated to be 30 , we randomly selected one class at each of grades one to six of every chosen school, and all students of that particular class were enrolled in the study. Thus, all students of 36 classes (each class as a cluster) were selected for the study. Parents were notified in advance through a letter regarding the screening their children for MHP. They were provided with a participant information leaflet about the study, emphasizing that it is not obligatory to participate in this study. They were then asked to sign and return the enclosed consent form, if they were willing for their child to participate. Researchers also informed parents about the aims of the study via briefing sessions at the schools, and provided instruction on completing the questionnaires. All questionnaires were anonymized, but had a specific confidential code for follow up purposes and contacting parents if necessary. The assessment tools for the study consisted of two parts. First part was the Persian version of SDQ Parent Report, which is used in children and ado- lescents. Psychometric features of this questionnaire have been evaluated in several studies in the Iranian population, and its validity and reliability have been confirmed in previous studies (4-6). The scale has five subscales including conduct problems, hyperactivity-inattention problems, emotional symptoms, peer problems and pro-social behaviors. Each subscale in turn consists of five items; and each item can be answered as "not true", "somewhat true" or "certainly true". "Somewhat true" is scored as 1, "not true" and "certainly true" can be scored o or 2 depending on each item. For each subscale the score ranges from 0 10. The score of all subscales (except pro-social behavior) is added up to generate a total score ranging from 0 - 40 . Scores 0 - 13 are considered as normal, 14 - 16 as borderline and 17 - 40 as abnormal. For pro-social behaviors' scores of 6 - 10 are considered normal, 5 is considered borderline, and 0 - 4, abnormal. The scale also included an Impact Supplement consisting of overall distress items and social impairment. The scores of items on overall distress and social impairment are added up to generate an impact score of 0 -10. A total impact score of 2 or above is abnormal. Second part, a demographic, social and cultural questionnaire, consisted of characteristics of the child including sex, age, type of school, birth rank, number of siblings, average monthly income and its relative adequacy, any significant physical illness or previously diagnosed psychiatric disorders in the child and other family members, the child's and the parents' living status (i.e. whether the child lived with one or both parents), the parent (s) employment and education level (i.e. up to 12 years old: high school level or higher: university level), and the time spent with the child by parent (s) per day. We used two partly related questions to evaluate economic status of child's family: Average amount (or mean) of monthly income (according to respondents' self-estimation and self-repot: $\geq 7000,000$ or $<$ 7000,000 Rials/month), and how adequate it was? (According to respondents' judgment: Not at all, somewhat, and sufficient). These factors were included on the basis of similar studies, and bearing in mind the specific aims of the study. The validity and reliability of the questionnaire was confirmed by a pilot study on 20 parents (Cronbach's alpha 0.731). For analysis we used free portable package of Stata-nine as epidemiologic statistical software, applying its survey related commands. We adjusted cluster effects in all point and interval estimations of prevalence, as well as population weights regarding the true proportions of population in subgroups defined according to child's gender (male/female), grade (one to six) and type of school (public/private). Associations between each socio-familial factor and MHP were assessed by fitting simple and multiple logistic regression models and their magnitude were reported via crude and adjusted Odds Ratios (OR). To fit regression models we divided all children into two main groups: abnormal (scores 17 - 40) and borderline/normal (scores 0 -16) according to total difficulties score, set as outcome (binomial dependent) variable in regression models. P values $<0.05$ were considered significant. 
Seyf Hashemi M et al.

Table 1. Demographic, Familial and Social Characteristics of the Participants (Number and Percentage of Missing Data on Each Item are not Shown) ${ }^{\mathrm{a}}$

\begin{tabular}{|c|c|}
\hline Characteristics & No. $(\%)$ \\
\hline \multicolumn{2}{|l|}{ Gender } \\
\hline Female & $584(48.7)$ \\
\hline Male & $616(51.3)$ \\
\hline \multicolumn{2}{|l|}{ Grade } \\
\hline 1 & $219(18.3)$ \\
\hline 2 & $211(17.6)$ \\
\hline 3 & $197(16.4)$ \\
\hline 4 & $194(16.2)$ \\
\hline 5 & $193(16.1)$ \\
\hline 6 & $186(15.5)$ \\
\hline \multicolumn{2}{|l|}{ Type of School } \\
\hline Public & $1023(85.3)$ \\
\hline Private $^{\mathrm{a}}$ & $177(14.7)$ \\
\hline \multicolumn{2}{|l|}{ First child } \\
\hline Yes & $647(54.0)$ \\
\hline No & $550(46.0)$ \\
\hline \multicolumn{2}{|c|}{ Diagnosed physical illness in child } \\
\hline Yes $^{b}$ & $23(1.9)$ \\
\hline No & $1161(98.1)$ \\
\hline \multicolumn{2}{|c|}{ Previously diagnosed psychiatric disorders } \\
\hline Yes $^{\mathrm{C}}$ & $34(2.9)$ \\
\hline No & $1132(97.1)$ \\
\hline \multicolumn{2}{|c|}{ Number of siblings } \\
\hline 1 & $262(21.9)$ \\
\hline$>1$ & $931(78.1)$ \\
\hline \multicolumn{2}{|c|}{ Disorders in other family members } \\
\hline Yes $^{\mathrm{d}}$ & $44(3.8)$ \\
\hline No & $1111(96.2)$ \\
\hline \multicolumn{2}{|c|}{ History of death of siblings } \\
\hline Yes & $51(4.5)$ \\
\hline No & $1096(95.5)$ \\
\hline \multicolumn{2}{|c|}{ Living with both Parents } \\
\hline Yes & $1162(97.4)$ \\
\hline No & $31(2.6)$ \\
\hline \multicolumn{2}{|c|}{ Average income, 10,000 Rials/month } \\
\hline$<700$ & $703(58.6)$ \\
\hline$\geq 700$ & $456(38.0)$ \\
\hline \multicolumn{2}{|c|}{ Income relative adequacy } \\
\hline Not at all & $96(8.2)$ \\
\hline Somewhat & $839(71.7)$ \\
\hline Sufficient & $237(20.2)$ \\
\hline \multicolumn{2}{|l|}{ Mother's job } \\
\hline Employed & $420(35.5)$ \\
\hline Housewife & $762(64.5)$ \\
\hline \multicolumn{2}{|c|}{ Education of mother, $y$} \\
\hline$\leq 12$ & $645(55.0)$ \\
\hline$>12$ & $527(45.0)$ \\
\hline \multicolumn{2}{|c|}{ Time spent with the child by mother, $d$} \\
\hline$\leq 2 \mathrm{~h}$ & $158(13.4)$ \\
\hline$>2 \mathrm{~h}$ & $1020(86.6)$ \\
\hline \multicolumn{2}{|l|}{ Father's job } \\
\hline Employed & $574(52.4)$ \\
\hline Self-employed & $495(45.2)$ \\
\hline Unemployed & $25(2.3)$ \\
\hline \multicolumn{2}{|c|}{ Education of father, $y$} \\
\hline$\leq 12$ & $575(49.4)$ \\
\hline$>12$ & $589(50.6)$ \\
\hline
\end{tabular}

\begin{tabular}{|c|c|}
\hline$\leq 2 \mathrm{~h}$ & $713(61.5)$ \\
\hline$>2 \mathrm{~h}$ & $446(38.5)$ \\
\hline \multicolumn{2}{|c|}{ Death of parents } \\
\hline Yes & $15(1.3)$ \\
\hline No & $1185(98.7)$ \\
\hline \multicolumn{2}{|c|}{$\begin{array}{l}\mathrm{a} \text { Tuition fee required. } \\
\mathrm{b} \text { Asthma or allergies, anemia, cardiovascular disorders, hearing problems, } \\
\text { visual problems, urologic problems, diabetes and chronic constipation, as } \\
\text { reported by the respondent. } \\
\text { c Including behavioral, depressive and anxiety disorders. } \\
\mathrm{d} \text { As reported by the respondent including significant disorders such as } \\
\text { cardiovascular disease and stroke, mental illness, diabetes, malignancies, } \\
\text { and various anomalies and disabilities. }\end{array}$} \\
\hline
\end{tabular}

\section{Results}

We gave the questionnaire to 1254 students in 36 classes and received 1200 completed questionnaires from parents (response rate more than 95\%). With regard to sex distribution, our sample consisted of 584 (48.7\%) girls and 616 (51.3\%) boys. The results showed that $23(1.9 \%)$ children had a known physical health problem, while 34 (2.9\%) children had a previously established diagnosis of mental health condition including behavioral, depressive and anxiety disorders. The demographic, familial and social characteristics of all participants are shown in Table 1. Total scores of 967 children (80.6\%) were within normal range. After population adjusting, 9.6\% (95\% CI: $3.1,16.1)$ of children scored in the abnormal and $9.7 \%$ ( $95 \%$ CI: $5.5,14.0$ ) scored in the borderline bands. In other words, $19.3 \%$ of the children scored above the normal threshold. In terms of gender, boys were affected more (13.3\%) than twice as much as girls (5.6\%). With regard to SDQ subscales, the frequency of problems ranged between $16.1 \%$ (peer problems) and 8.4\% (emotional symptoms); and across all subscales, the percentage of affected Boys was nearly twice as much as affected girls. Also, girls scored higher than boys for pro-social behaviors, indicating better pro-social behaviors in girls. The impact score was abnormal in $68.4 \%$ (95\% CI: $59.9,76.9)$ of all children, with girls scoring more than boys (72.0\% vs. $65.0 \%$ ). The population adjusted point and interval prevalence of MHP across the five different subscales and in total, and the impact of these MHP are shown in Table 2. The study also assessed the association between certain important personal, familial and social characteristics of the participants with abnormal SDQ score (Table 3), as seen in Table 3, the findings showed a significant and high magnitude association between an abnormal SDQ total score and a previously diagnosed mental health disorder in children. Moreover, crude (prior to adjusting) OR for male gender, health problems in family members, housewife mothers, unemployed fathers, low family income, and less time spent with the child by parents were also significantly associated with an abnormal SDQ total scores in children. In addition, higher educational level of parents was significantly associated with a lower chance of having abnormal SDQ total score. After adjusting for all factors, a previously diagnosed mental health disorder $(\mathrm{OR}=11.11,95 \% \mathrm{CI}$ : 5.55, 
25.00) and male gender ( $\mathrm{OR}=1.43,95 \% \mathrm{CI}: 1.10,1.87)$ were still significantly associated with an abnormal SDQ total score. Less time spent with the child by father was also associated with a higher chance of abnormal SDQ total score $(\mathrm{OR}=1.61,95 \% \mathrm{CI}: 1.20,2.17)$. In addition, there was a considerable (but not significant) association between studying in public school with abnormal SDQ score in terms of adjusted $\mathrm{OR}(\mathrm{OR}=1.54, \mathrm{P}=0.06)$.

Table 2. Population Adjusted Prevalence of Mental Health Problems (MHP) According to Strengths and Difficulties Questionnaire (SDQ) in the Urban Population of Elementary School Students in Semnan by Gender, 2012 a

\begin{tabular}{|c|c|c|c|}
\hline \multirow[t]{2}{*}{ MHP Status (Corresponding Scores) } & \multicolumn{3}{|c|}{ Prevalence (95\% CI) } \\
\hline & Male & Female & Total \\
\hline \multicolumn{4}{|l|}{ Total difficulties } \\
\hline Normal (0 - 13) & $75.1(65.0,85.2)$ & $86.4(84.2,88.6)$ & $80.6(69.9,91.3)$ \\
\hline Borderline (14 - 16) & $11.5(6.7,16.2)$ & $7.9(5.5,10.4)$ & $9.7(5.5,14.0)$ \\
\hline Abnormal (17 - 40) & $13.4(8.0,18.8)$ & $5.6(5.5,5.8)$ & $9.6(3.1,16.1)$ \\
\hline \multicolumn{4}{|l|}{ Emotional } \\
\hline Normal (0 - 3) & $82.6(78.3,86.8)$ & $88.01(80.4,95.5)$ & $85.2(78.2,92.1)$ \\
\hline Borderline (4) & $6.9(5.9,7.9)$ & $5.6(0.25,11.6)$ & $6.3(2.9,9.7)$ \\
\hline Abnormal $(5-10)$ & $10.4(5.4,15.4)$ & $6.3(4.6,7.9)$ & $8.4(3.8,12.9)$ \\
\hline \multicolumn{4}{|l|}{ Hyper activity } \\
\hline Normal (0 - 5) & $68.7(61.2,76.1)$ & $79.4(78.3,80.5)$ & $73.9(64.7,83.1)$ \\
\hline Borderline (6) & $11.8(7.4,16.2)$ & $10.1(8.04,12.3)$ & $11.03(7.9,14.1)$ \\
\hline Abnormal $(7-10)$ & $19.4(15.6,23.2)$ & $10.4(9.3,11.4)$ & $15.04(8.3,21.7)$ \\
\hline \multicolumn{4}{|l|}{ Conduct } \\
\hline Normal (0 - 2) & $71.8(62.7,80.9)$ & $80.5(84.4,85.5)$ & $78.9(66.4,91.5)$ \\
\hline Borderline (3) & $13.4(10.2,16.5)$ & $7.2(5.7,8.7)$ & $10.4(5.2,15.5)$ \\
\hline Abnormal $(4-10)$ & $14.7(8.2,21.2)$ & $6.2(5.7,6.7)$ & $10.6(3.08,18.1)$ \\
\hline \multicolumn{4}{|l|}{ Peer } \\
\hline Normal (0 - 2) & $59.9(43.9,76.02)$ & $68.9(64.4,73.5)$ & $64.3(52.4,76.2)$ \\
\hline Borderline (3) & $19.2(15.5,23.04)$ & $19.7(18.7,20.6)$ & $19.4(17.4,21.4)$ \\
\hline Abnormal (4 - 10) & $20.7(8.3,33.1)$ & $11.3(5.8,16.8)$ & $16.1(5.1,27.1)$ \\
\hline \multicolumn{4}{|l|}{ Pro-social } \\
\hline Normal (6-10) & $90.9(85.06,96.9)$ & $93.9(92.6,95.2)$ & $92.4(88.6,96.2)$ \\
\hline Borderline (5) & $4.9(2.3,7.5)$ & $3.9(2.9,4.8)$ & $4.4(2.8,6.04)$ \\
\hline Abnormal (0-4) & $4.1(0.5,7.5)$ & $2.1(1.7,2.4)$ & $3.1(0.8,5.4)$ \\
\hline \multicolumn{4}{|l|}{ Impact } \\
\hline $\operatorname{Normal}(0)$ & $30.3(24.2,36.4)$ & $25.8(19.1,32.4)$ & $28.1(21.5,34.6)$ \\
\hline Borderline (1) & $4.6(3.9,5.3)$ & $2.2(0.4,3.9)$ & $3.5(1.3,5.7)$ \\
\hline Abnormal (2-10) & $65.0(59.6,70.5)$ & $72.0(63.6,80.4)$ & $68.4(59.9,76.9)$ \\
\hline
\end{tabular}

a Abbreviation: CI, Confidence Interval. 
Table 3. Crude and Adjusted Odds Ratios of the Relationship Between Students Characteristics and Abnormal (17 - 40) Total Difficulties Score of Strengths and Difficulties Questionnaire (SDQ) ${ }^{\text {a }}$

\begin{tabular}{|c|c|c|c|c|}
\hline Characteristic & Crude OR $(95 \% \mathrm{CI})$ & PValue & Adjusted (OR(95\%CI) & P Value \\
\hline Male gender & $2.10(1.35-3.24)$ & 0.001 & $1.43(1.10-1.87)$ & 0.007 \\
\hline Grade & $0.97(0.92-1.2)$ & 0.3 & $0.98(0.93-1.02)$ & 0.4 \\
\hline Study in public school & $1.36(0.72-2.54)$ & 0.3 & $1.54(0.97-2.45)$ & 0.06 \\
\hline Birth rank & $1.03(0.87-1.22)$ & 0.7 & $0.97(0.65-1.44)$ & 0.9 \\
\hline Number of siblings & $1.17(0.95-1.45)$ & 0.1 & $1.07(0.7-1.64)$ & 0.7 \\
\hline Physical illness in child & $1.78(0.79-4.00)$ & 0.2 & $1.66(0.79-3.57)$ & 0.2 \\
\hline Psychiatric disorder in child & $11.11(7.69-16.66)$ & $<0.001$ & $11.11(5.55-25.00)$ & $<0.001$ \\
\hline Disease in Family members & $7.69(0.21-0.52)$ & $<0.001$ & $1.63(0.82-3.22)$ & 0.1 \\
\hline Education of mother (>12 years) & $0.48(1.92-4.76)$ & $<0.001$ & $1.03(0.60-1.77)$ & 0.9 \\
\hline Housewife mother & $1.97(1.22-3.17)$ & 0.005 & $1.23(0.78-1.93)$ & 0.4 \\
\hline Time spent with child by mother $\leq 2 \mathrm{~h} / \mathrm{d}$ ) & $1.39(0.92-2.32)$ & 0.1 & $1.67(0.94-2.00)$ & 0.08 \\
\hline Education of father ( $>12$ years) & $0.71(0.60-0.85)$ & $<0.001$ & $0.66(0.42-1.05)$ & 0.5 \\
\hline Unemployed father & $1.56(1.24-1.97)$ & $<0.001$ & $1.22(0.76-1.94)$ & 0.4 \\
\hline Time spent with child by father $\leq 2 \mathrm{~h} / \mathrm{d}$ ) & $2.00(1.75-2.27)$ & $<0.001$ & $1.61(1.20-2.17)$ & 0.002 \\
\hline Not living with both parents & $1.52(0.82-2.7)$ & 0.2 & $1.87(0.10-34.9)$ & 0.7 \\
\hline History of death of siblings & $1.26(0.54-2.91)$ & 0.6 & $1.24(0.46-2.31)$ & 0.5 \\
\hline Income ( $\geq 7000,000$ Rials/month) & $0.67(0.48-0.93)$ & 0.02 & $0.87(0.57-1.31)$ & 0.5 \\
\hline Income adequacy $^{\mathrm{b}}$ & $0.86(0.77-0.96)$ & 0.009 & $1.37(0.96-1.95)$ & 0.08 \\
\hline
\end{tabular}

a Abbreviations: CI, Confidence Interval; OR, Odds Ratios.

b Not at all: 1, somewhat: 2, sufficient: 3 .

\section{Discussion}

The first and most important step in the prevention and management of MHP is to find the extent of these problems in children and adolescents at the community level. In the current study the parent-rated SDQ was used to assess MHP in a representative sample of children and adolescents. The Persian version of SDQ had already been validated in the Iranian population $(6,7)$, and its reliability as a psychometric tool demonstrated in several population based studies $(4,5)$. The present study assessed SDQ data set obtained from 1200 primary school children aged 6 - 12 years old. The findings revealed that the proportion of children with a high risk of MHP was 9.6\%, and for those with a borderline risk, 9.7\%. Several factors including male gender, physical health problems in family members, housewife mothers, low educated mothers, unemployed and low educated fathers, and low family income were marginally associated with a higher chance of occurrence of MHP in children and adolescents. The study showed that based on parent report SDQ, 9.6\% of children had total scores above 17, and that among these, peer problems were the most frequent difficulty. These findings are considerably lower than those of a similar Iranian study conducted 4 years earlier in Tehran (8) in which the total SDQ score of $25.8 \%$ of children in the same age range was higher than 17 . In the Tehran study conduct problem was the most frequent problem, followed by

peer problems (8). This rate was $26 \%$ in Arman's study carried out in Isfahan using the same research tools and method (9). Isfahan's study showed conduct problems and peer problems to be the most frequent difficulties in children (9). The result of the present study was in agreement with those of several European studies which used the same tool. The proportion of children classified as borderline or abnormal based on parent rated SDQ has been $18.5 \%$ in 3 - 17 year-old German children (10) which is consistent with the result of our study (19.3\%). In a comparative study in the UK the frequency of MHP in white British children and refugee children was $15 \%$ and $27 \%$, respectively (11); while in another study using ICD 10 diagnostic criteria, $10 \%$ of British children and adolescents aged 5 - 15 suffered from MHP (12). In the BELLA study (13) performed on a large population in Germany using SDQ, $16.1 \%$ of $7-11$ year-olds scored above the normal range (borderline or abnormal score) which is slightly lower than our result (19.3\%). In Nordic countries the frequency of MHP according to SDQ parent-report has been much lower than in other countries, ranging between 5.7\% and 7.2\% (14). Among other countries of the region, $34.4 \%$ of children aged 5 - 11 in Pakistan (15) have scored higher than borderline (total score $>17$ ). This rate has been $14 \%$ in Arab children in the Gaza strip (16); with both of these being much higher than that of the present study in Iran (9.6\%). 
In a study on 3 - 17 year-old children in China, $21 \%$ of children scored higher than normal ( $8.9 \%$ borderline and $12.1 \%$ abnormal) (17). The present study revealed the prevalence of MHP in children in an Iranian urban community sample to be less than Pakistan, China and Gaza strip; and higher than England, Germany and the Nordic countries. These findings predictably reflect such differences as socioeconomic and cultural parameters, as well as in the availability and extent of mental health care in these countries. The most frequent problem in the present study was peer problems followed by hyperactivity problems; while in certain other countries such as the Nordic countries, Britain and China it was hyperactivity problems followed by emotional problems, and in Germany, conduct problems. In our study boys scored higher than girls in all SDQ subscales, which is in agreement with the results of similar studies in Britain, Germany, Denmark, China and Pakistan, demonstrating that boys are at a higher risk of MHP compared to girls. With regard to the impact of MHP on various aspects of children and their families' life, $68.4 \%$ of children had an abnormal impact score. Despite scoring very high in the impact supplement, many of these children were not identified by SDQ screening and their total score did not indicate a mental health problem (scored less than 13). The rate of abnormal impact score was higher in girls than in boys. Although $13.3 \%$ of boys had an abnormal total SDQ score, $65 \%$ of them had abnormal impact scores. Interestingly however; although total SDQ score in girls was abnormal in $5.6 \%$ of girls, $72 \%$ of them had abnormal impact scores. The impact score in our study was much higher than those of other countries. In the BELLA study $12 \%$ of children with abnormal impact score were found to have normal SDQ total score (18). In the Copenhagen cohort study, only $4.8 \%$ of children had an abnormal impact score (19), while a screening for psychopathology in child welfare in Belgium showed that $49 \%$ of children in child welfare had an abnormal impact score (20); which is still less than the rate in our study. In developed countries, the extensive state support of families who have children with MHP is likely to reduce the burden caused by these problems and can explain the lower rate of impact compared to developing countries like Iran. The high rate of children with abnormal impact scores in our study may also reflect the significant sensitivity of parents toward their children's feelings and behavior. Some children had abnormal scores in only a few items, but their parents reported a high or very high impact of the reported problems on educational and social aspects of the children's life. Although the proportion of girls who had abnormal total SDQ scores was approximately less than half of the boys with abnormal total score (5.6\% compared to $13.3 \%$ ), the percentage of families who were affected by these problems was higher in girls than in boys (72\% compared to $65 \%)$.This reflects the greater sensitivity of parents toward their daughters' behavioral and emotional problems as compared to their sons'; a finding which is cultur- ally justifiable in most Iranian families. Likewise, psychiatric problems in girls may be more likely to cause personal distress, emotional expression and family impairment compared to boys. Among participants in the study, $2.9 \%$ of children had a previously diagnosed mental health problem. According to the parent report version of SDQ 19.3\% of participants scored above the normal threshold. There was a significant association between having a diagnosed mental health problem and abnormal SDQ total score $(\mathrm{OR}=11.11,95 \% \mathrm{CI}=5.55-25.00, \mathrm{P}<$ 0.001) which further underpins the validity of SDQ. Other variables significantly associated with abnormal total score of SDQ in simple marginal analyses were: male gender, physical health problems in family members, low level of mother and father's education, housewife mothers, unemployed fathers, and low family income. Some of these correlations are in line with other studies. In the Copenhagen study (19) the prevalence of MHP was higher among children with low level of maternal education. This finding was also reported in a prevalence study in Italy (21). The results of the study by Arman et al. showed that abnormal SDQ total scores in adolescents were associated with lower level of education in both mothers and fathers (9). Low family income has been correlated with a higher risk of MHP in all these three surveys, which is well-matched with our results. In another prevalence study in Great Britain (12) the rate of MHP was greater among children of parents without educational qualifications than highly educated parents (15\% vs. $6 \%$ ) and in families with neither parent working, compared to both parents being at work ( $20 \%$ vs. $8 \%$ ). All of these findings are in line with the results of the present study. The fact that parents with low level of education are likely to have a lesser degree of knowledge, and hence overall insight about dealing with their children's behavioral and emotional problems, can largely explain this correlation. Great levels of emotional and behavioral problems were reported in our study in children of unemployed fathers and also parents who spent less time with their children. Although unemployed fathers may spend more hours with their children, financial problems and low self-confidence arising from unemployment usually can interfere with having a good relationship with children. These findings underpin the fact that social and economic disadvantages can increase the risk of MHP in children and adolescents.

\subsection{Strengths and Limitations}

Our participants are highly representative of 6 - 12 year old children in the urban population of interest, as they were recruited from primary schools, and as school attendance is mandatory for this age group in Iran with a rate of $99.76 \%$ (22). Other strengths of our study are the large number of participants, low level of unresponsiveness, and the use of SDQ for screening, which is a short and simple tool with a very high sensitivity and specific- 
ity. The most important limitation of the present study is that we did not use teacher and self-versions of this questionnaire which may limit and interfere with the reliability of the results. The high rate of behavioral and emotional problems of children, and the great impairment arising from these problems underpin the importance of screening for MHP in children and adolescents using standard and simple screening tools. As there was no significant correlation between the age of children and abnormal SDQ total scores, we recommend introducing MHP screening in schools at an early stage, with particular focus on high risk groups.

\section{Authors' Contributions}

Maryam Seyf Hashemi: concept, design, interpretation, drafting of the manuscript, critical revision of the manuscript and approval of the article. Elham Yarian: design, acquisition of data, interpretation, drafting of the manuscript. Parviz Bahadoran: drafting of the manuscript, critical revision of the manuscript and approval of the article. Jafar Jandaghi: acquisition of data, drafting of the manuscript. Majid Mirmohammad Khani: design, data analysis, interpretation, critical revision of the manuscript and approval of the article.

\section{References}

1. World Health Organization . Mental health: a state of well-being. WHO; [cited Dec 7, 2013]. 2014. Available from: http://www.who. int/features/factfiles/mental_health/en/

2. Child mental health and human capital accumulation: the case of ADHD. Currie J, Stabile M. J Health Econ. 2006;25(6):1094.

3. Prevalence, severity, and unmet need for treatment of mental disorders in the World Health Organization World Mental Health Surveys. Demyttenaere K, Bruffaerts R, Posada-Villa J, Gasquet I, Kovess V, Lepine JP, et al. JAMA. 2004;291(21):2581.

4. Normative data and psychometric properties of the parent and teacher versions of the strengths and difficulties questionnaire (SDQ) in an Iranian community sample. Shahrivar Z, Tehrani-Doost M, Pakbaz B, Rezaie A, Ahmadi F. J Res Med Sci. 2009;14(2):69.

5. Psychometric Properties of Persian Parent and Teacher Versions of the Strengths and Difficulties Questionnaire in a Sample of Iranian Children. Aguilar-Vafaie M, Gharehbaghy F. Iran J Psychiatry Clin Psychology. 2009;15(3):231.

6. Scale validation of the strengths and difficulties questionnaire in Iranian children. Ghanizadeh A, Izadpanah A, Abdollahi G. Iran J Psychiatry. 2007;2(2):65.

7. Frequency of Psychological Disorders amongst Children in Urban Areas of Tehran. Alavi A, Mohammadi MR, Joshaghani N, Mahmoudi-Gharaei J. Iran J Psychiatry. 2010;5(2):55.
8. Children Mental Health Problems: Parent Report Form of Strength and Difficulties Questionnaire. Mohammadi MR, Alavi A, Mahmoudi-Gharaei J, Tehranidoost M, Shahrivar Z, Saadat S. Iran J Psychiatry. 2008;3(1):26.

9. Epidemiological Study of Youth Mental Health Using Strengths and Difficulties Questionnaire (SDQ). Arman S, Keypour M, Maracy MR, Attari A. Iran Red Crescent Med J. 2012;14(6):371.

10. Assessing psychopathological problems of children and adolescents from 3 to 17 years in a nationwide representative sample: results of the German health interview and examination survey for children and adolescents (KiGGS). Holling H, Kurth BM, Rothenberger A, Becker A, Schlack R. Eur Child Adolesc Psychiatry. 2008;17 Suppl 1:34-41.

11. Mental health of refugee children: comparative study. Fazel M, Stein A. BMJ. 2003;327(7407):134.

12. Mental health of children and adolescents in Great Britain. Meltzer H, Gatward R, Goodman R, Ford T. Int Rev Psychiatry. 2003;15(1-2):185.

13. The mental health module (BELLA study) within the German Health Interview and Examination Survey of Children and Adolescents (KiGGS): study design and methods. Ravens-Sieberer U, Kurth BM, Ki GG, Bella study group Eur Child Adolesc Psychiatry. 2008;17 Suppl 1:10-21.

14. The Strengths and Difficulties Questionnaire in the Nordic countries. Obel C, Heiervang E, Rodriguez A, Heyerdahl S, Smedje H, Sourander A, et al. Eur Child Adolesc Psychiatry. 2004;13 Suppl 2:II32-9.

15. Screening for emotional and behavioural problems amongst 5-11-year-old school children in Karachi, Pakistan. Syed EU, Hussein SA, Mahmud S. Soc Psychiatry Psychiatr Epidemiol. 2007;42(5):421.

16. Child mental health problems in Arab children: application of the strengths and difficulties questionnaire. Thabet AA, Stretch D, Vostanis P. Int J Soc Psychiatry. 2000;46(4):266.

17. The validity, reliability and normative scores of the parent, teacher and self report versions of the Strengths and Difficulties Questionnaire in China. Du Y, Kou J, Coghill D. Child Adolesc Psychiatry Ment Health. 2008;2(1):8.

18. How impaired are children and adolescents by mental health problems? Results of the BELLA study. Wille N, Bettge S, Wittchen HU, Ravens-Sieberer U, Bella study group Eur Child Adolesc Psychiatry. 2008;17 Suppl 1:42-51.

19. The prevalence of SDQ-measured mental health problems at age 5-7 years and identification of predictors from birth to preschool age in a Danish birth cohort: the Copenhagen Child Cohort 2000. Elberling H, Linneberg A, Olsen EM, Goodman R, Skovgaard AM. Eur Child Adolesc Psychiatry. 2010;19(9):725.

20. Screening for psychopathology in child welfare: the Strengths and Difficulties Questionnaire (SDQ) compared with the Achenbach System of Empirically Based Assessment (ASEBA). Janssens A, Deboutte D. Eur Child Adolesc Psychiatry. 2009;18(11):691.

21. Prevalence and correlates of mental disorders among adolescents in Italy: the PrISMA study. Frigerio A, Rucci P, Goodman R, Ammaniti M, Carlet O, Cavolina P, et al. Eur Child Adolesc Psychiatry. 2009;18(4):217.

22. [cited Dec 7, 2013];Index Mundi Iran-School enrollment. Available from: http://www.indexmundi.com/facts/iran/school-enrollment. 